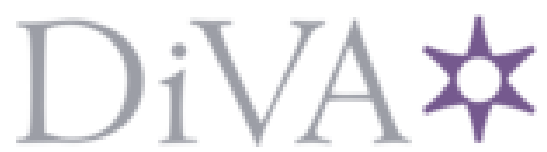

http://www.diva-portal.org

Postprint

This is the accepted version of a paper presented at 2020 IEEE Power \& Energy Society Innovative Smart Grid Technologies Conference (ISGT), The Hague, The Netherlands, 25-28 October 2020.

Citation for the original published paper:

Avula, R R., Oechtering, T J. (2020)

Optimal privacy-by-design strategy for user demand shaping in smart grids In: Washington, DC, USA, USA

N.B. When citing this work, cite the original published paper.

Permanent link to this version:

http://urn.kb.se/resolve?urn=urn:nbn:se:kth:diva-271979 


\title{
Optimal privacy-by-design strategy for user demand shaping in smart grids
}

\author{
Ramana R. Avula and Tobias J. Oechtering \\ School of Electrical Engineering and Computer Science \\ KTH Royal Institute of Technology, Stockholm, Sweden
}

\begin{abstract}
In this work, we propose an optimal privacy-bydesign strategy using an energy storage system (ESS) that is capable of shaping the user demand to follow a time-varying target profile. In addition, we consider the ESS usage cost due to its energy losses and capacity degradation. We measure the privacy leakage in terms of the Bayesian risk. The proposed strategy is computed by solving a multi-objective optimization problem using the Markov decision process framework. With numerical simulations using real household consumption data and a lithium-ion battery model, we study the trade-off between the achievable Bayesian risk, the variations in the user demand from the target profile and the energy storage cost. The results show that by trading-off some privacy, the variations in the user demand can be reduced while improving the battery lifetime.
\end{abstract}

Index Terms-Smart meter privacy, energy flow management, Markov decision process, dynamic programming, Bayesian hypothesis testing, user demand shaping.

\section{INTRODUCTION}

Smart meters (SMs) are crucial components of smart grids which rely on the timely updates of the energy consumption data of the users in the network. Using the near real-time information about the user demands, the smart grids can better control the grid fluctuations and increase the overall system efficiency by a better forecast of the future energy demands of the users. However, with the advent of the nonintrusive appliance load monitoring (NILAM) techniques [1], the consumers in smart grids are prone to privacy risks as they enable a malicious adversary to infer the users' behaviour. In Europe, the General Data Protection Regulation (GDPR) defines strict rules to deal with data that contain sensitive personal information such as, health status, religious beliefs etc. This means that using SM data, one should not be able to infer presence of appliances or their usage patterns that may reveal sensitive private information.

Several privacy-preserving approaches have been proposed in the literature [2] which use an energy storage system (ESS) to alter the user demand from the grid in real-time, as shown in Fig. 1. In [3], a best-effort (BE) heuristic algorithm is proposed to hide the appliance usage information by resisting the user demand changes to the extent possible at each time instance. In [4]-[6] more heuristic algorithms are proposed. In [7], the privacy leakage is measured in terms of the Bayesian risk of an informed adversarial hypothesis testing model and the optimal strategy is designed which minimizes the cumulative risk over an infinite horizon using the partially observable

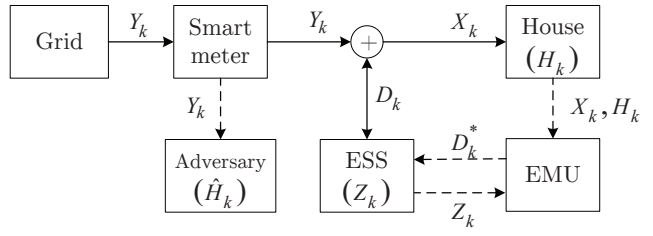

Fig. 1. The smart-metering system that shapes the user demand using an energy storage system. The solid lines here represent the power flow and the dotted lines represent the information flow.

Markov decision process (POMDP) framework. In [8], the authors presented the optimal privacy-cost trade-off with the time-varying electricity price by measuring privacy in terms of variations in the load compared to a fixed-target value using a lossless ESS model. In [9]-[11] the Bayesian risk is minimized while taking into account the ESS aspects such as the energy losses and capacity degradation using detailed ESS models. In contrast to the previous work, here we leverage the energy storage capacity of the smart-metering system. While considering the Bayesian risk and the ESS usage cost due to its energy losses and capacity degradation, we present an optimal energy-flow control strategy that minimizes the variations in the user demand from a time-varying target profile. The proposed privacy-by-design strategy can be used in applications such as grid load balancing, energy arbitrage in electricity markets etc, with privacy as a secondary objective. With extensive numerical simulations, we evaluate the tradeoff between the minimum achievable Bayesian risk and the variations in the user demand.

The rest of the paper is organized as follows. In Section II, we present an overview of the privacy-preserving smartmetering system, and its model assumptions. In Section III, we present the design of the optimal EMU strategy. In Section IV, we discuss the results of a numerical study and lastly, we conclude the paper in Section V. Throughout the paper, we denote random variables by the capital letters, their realizations by the lower-case letters, and their alphabets by the calligraphic letters. We use the notation $A_{k}^{k+i}$ to denote the row vector $\left[A_{k}, A_{k+1}, \ldots, A_{k+i}\right] ; \mathbb{E}[\cdot]$ to denote the expectation operator; $(\cdot)^{\top}$ to denote the matrix transpose operator; $P_{A}(a)$ to denote a probability distribution function; $\Delta_{n}$ to denote an $(n-1)$ dimensional simplex space; and $\mathcal{B}(\mathcal{A})$ to denote the set of all finite real-valued functions on discrete or continuous space $\mathcal{A}$. In the equations, if the domain of a variable is not specified in summations, then it is summed over its domain. 


\section{SYSTEM OVERVIEW}

We consider the smart-metering system as shown in Fig. 1. The energy consumption measured by the SM is shaped by an EMU using a strategy for the discrete-time finite-horizon $\mathcal{K}:=\{1,2, \ldots, N\}$. Within $\mathcal{K}$, let $k$ denote the index of the time slot with a fixed time duration $T$. At the beginning of each slot $k$, the EMU strategy specifies the charge or discharge power level of the ESS. We consider discrete quantized power and energy measurements with $w$ and $w T$ as their respective resolutions. Further, we assume a maximum average power demand of the user, denoted by $x_{\max }$. We consider an ESS with a finite rated energy capacity denoted by $z_{\max }$. Using the convention that positive ESS power means charging, we denote the rated discharge and charge powers by $d_{\mathrm{r}, \mathrm{d}}$ and $d_{\mathrm{r}, \mathrm{c}}$ respectively, where $d_{\mathrm{r}, \mathrm{d}}<0$ and $d_{\mathrm{r}, \mathrm{c}}>0$. We use $y_{\max }$ to denote the maximum power measurable by the SM. For each $k \in \mathcal{K}$, we model the user power demand, the SM measurement, the operating ESS power and the energy state of the ESS using discrete random variables denoted by $X_{k}$, $Y_{k}, D_{k}$, and $Z_{k}$ respectively. Further, we model the events of user demands or user behaviour using a sequence of hypothesis states $H_{k}$ that should be protected. That is, the SM readings should reveal as little information about $H_{k}$ as possible. Let $h_{\max }$ denote the maximum number of possible hypothesis states. Table I summarizes the alphabets of these random variables. Let $S_{k}:=\left[X_{k}, Z_{k}, H_{k}, Y_{k-1}\right]$ denote the EMU state vector, which is defined in $\mathcal{S}=\mathcal{X} \times \mathcal{Z} \times \mathcal{H} \times \mathcal{Y}$.

At each time-slot $k$, the EMU aims to shape the user demand from the grid $Y_{k}$ to follow a target signal $\bar{Y}_{k}$ which is a discrete random variable defined on $\mathcal{Y}$. In this work, we design the EMU for the class of target signals $\bar{Y}_{k}$ that can be characterized by a conditional probability function $P_{\bar{Y}_{k} \mid S_{k}}$, which is either given by a grid operator to achieve demand side management or it can be set by the EMU to achieve any secondary objective. Further, we model the dependency between the random variables in the sequence $\left[H_{1}^{N}, X_{1}^{N}\right]$ using the Markov chains given by

$$
\begin{gathered}
H_{k}-H_{k-1}-\left(H_{1}^{k-2}, X_{1}^{k-1}\right), \\
X_{k}-H_{k}, X_{k-1}-\left(H_{1}^{k-1}, X_{1}^{k-1}\right) .
\end{gathered}
$$

Consequently, the joint probability distribution of $\left[H_{1}^{N}, X_{1}^{N}\right]$ is completely characterized by the parameter set $\theta$ given by

$$
\theta:=\left\{P_{H_{k} \mid H_{k-1}}, P_{X_{k} \mid H_{k}}, P_{H_{0}} \mid k \in \mathcal{K}\right\},
$$

where $P_{H_{k} \mid H_{k-1}}$ and $P_{X_{k} \mid H_{k}}$ denote the time dependent transition and emission probabilities, and $P_{H_{0}}$ denote the probability of the hypothesis state $H_{0}$ prior to the time-horizon $\mathcal{K}$. Further, we measure the privacy leakage using the Bayesian risk which is the average cost of an adversary making a guess on the hypothesis sequence. Let $\hat{\theta}$ denote the adversarial estimate of the HMM parameter set, $\hat{H}_{k}$ denote the adversarial guess, and $C_{h, \hat{h}}$ denote the Bayesian cost of adversarial guess $\hat{h}$ when the true hypothesis state is $h$. Let $\mathcal{R}_{k}$ denote the Bayesian risk, which is given by

$$
\mathcal{R}_{k}:=\mathbb{E}\left[C_{H_{k}, \hat{H}_{k}}\right]=\sum_{h, \hat{h}} C_{h, \hat{h}} \cdot P_{H_{k}, \hat{H}_{k}}(h, \hat{h}) .
$$

To simplify the analysis, we assume that the adversary is unaware of the presence of the ESS and makes a guess $\hat{H}_{k}$ using only the causal information available at time $k$. Further, we model the ESS using a memoryless stochastic model given in [11], which is characterized by a conditional probability distribution $P_{Z_{k+1}, E_{k}, B_{k} \mid D_{k}, Z_{k}}$ where $E_{k}, B_{k}$ denote the energy losses and capacity degradation of the ESS due to the EMU control actions. Let $L_{k}$ denote the expected ESS usage cost which is given by $L_{k}=\mathbb{E}\left[\rho B_{k}+\lambda_{k} E_{k}\right]$, where $\rho$ is the price (in $€$ ) of unit capacity loss and $\lambda_{k}$ is the price (in $€$ ) of unit energy. Let $f_{L}: \mathcal{Z} \times \mathcal{D}_{k} \rightarrow \mathbb{R}_{+}$denote the mean ESS usage cost which can be computed from the ESS model. Lastly, we define a weighted cost function of the expected deviation of the SM readings from the target signal, the Bayesian risk and the ESS usage cost, denoted by $\mathcal{C}_{k}$, given as

$\mathcal{C}_{k}:=\sigma[\underbrace{\omega \beta \mathbb{E}\left[\left|\bar{Y}_{k}-Y_{k}\right|\right]+(1-\omega) \gamma \mathcal{R}_{k}}_{\text {utility }}]+(1-\sigma) L_{k}$,

where $\sigma, \omega \in[0,1]$ are the trade-off parameters, $\beta, \gamma>0$ are the scaling factors of the objective functions. Here the weighted cost of the deviation and the Bayesian risk represent the utility of the EMU. In this work, we are interested in designing the optimal EMU that minimizes the aggregate weighted $\operatorname{cost} \mathcal{C}_{k}$ in the finite-horizon $\mathcal{K}$.

\section{OPtimal EnERgy MANAGEMENT UNIT STRATEGy}

In this section, we compute an optimal EMU strategy for the finite-horizon $\mathcal{K}$ using the MDP framework. Here, we provide only the key steps in the EMU strategy design and the detailed derivation can be done following the framework in [11]. Let $I_{k}:=S_{1}^{k}=\left[X_{1}^{k}, Z_{1}^{k}, H_{1}^{k}, Y_{1}^{k}\right]$ denote the set of all information available to the EMU at time-slot $k$. Further, let $\mu_{k}$ denote a randomized strategy of the EMU at time-slot $k$ that specifies a distribution of control action $D_{k}$ given $I_{k}$, i.e., $P_{D_{k} \mid I_{k}}$. Let $\mathcal{D}_{k}$ denotes the alphabet of $D_{k}$ satisfying the ESS operating constraints given in [11], $\mathcal{I}_{k}$ denote the alphabet of $I_{k}$, and $\mathcal{U}_{k}$ denotes the set of all mappings from $\mathcal{I}_{k}$ to $\Delta_{\left|D_{k}\right|}$. For a given strategy $\mu_{k} \in \mathcal{U}_{k}$ and for a given information $i_{k} \in \mathcal{I}_{k}$, the weighted cost $\mathcal{C}_{k}$ defined in (5) becomes

$$
\begin{gathered}
\mathcal{C}_{k}\left(i_{k}, \mu_{k}\right)=\sum_{d_{k}, \bar{y}_{k}} P_{D_{k} \mid I_{k}}\left(d_{k} \mid i_{k}\right) P_{\bar{Y}_{k} \mid S_{k}}\left(\bar{y}_{k} \mid s_{k}\right)\left[\sigma \omega \beta\left|\bar{y}_{k}-y_{k}\right|+\right. \\
\left.\sigma(1-\omega) \gamma \sum_{\hat{h}} C_{h_{k}, \hat{h}}\left[\bar{\zeta}^{*}\left(y_{1}^{k}\right)\right]_{\hat{h}}+(1-\sigma) f_{L}\left(z_{k}, d_{k}\right)\right]
\end{gathered}
$$

TABLE I

SUBSET OF SYSTEM VARIABLES

\begin{tabular}{|c|c|c|}
\hline Description & Variable & Alphabet or Domain \\
\hline User power demand & $X_{k}$ & $\mathcal{X}:=\left\{0, w, 2 w, \ldots, x_{\max }\right\}$ \\
\hline SM measurement & $Y_{k}$ & $\mathcal{Y}:=\left\{0, w, 2 w, \ldots, y_{\max }\right\}$ \\
\hline ESS power & $D_{k}$ & $\mathcal{D}:=\left\{d_{\mathrm{r}, \mathrm{d}}, \ldots,-w, 0, w, \ldots, d_{\mathrm{r}, \mathrm{c}}\right\}$ \\
\hline ESS energy state & $Z_{k}$ & $\mathcal{Z}:=\left\{0, w T, 2 w T, \ldots, z_{\max }\right\}$ \\
\hline Hypothesis state & $H_{k}$ & $\mathcal{H}:=\left\{1,2, \ldots, h_{\max }\right\}$ \\
\hline
\end{tabular}


where $y_{k}=x_{k}+d_{k}$, and $\bar{\zeta}^{*}$ denotes the optimal adversarial strategy to make a guess $\hat{H}_{k}$ given the SM measurements $y_{1}^{k}$ and the HMM $\hat{\theta}$. According to [11], a sufficient statistic for the computation of the optimal adversarial strategy $\bar{\zeta}^{*}$ is

$$
\hat{\pi}_{k}=f_{\hat{\pi}}\left(\hat{\pi}_{k-1}, y_{k}\right),
$$

where $f_{\hat{\pi}}: \Delta_{|\mathcal{H}|} \times \mathcal{Y} \rightarrow \Delta_{|\mathcal{H}|}$ is a linear fractional function that computes the belief state $\hat{\pi}_{k}$ using the HMM $\hat{\theta}$. Further, it is shown in [11] that the $\bar{\zeta}^{*}$ can be represented in the form of a set of polyhedral decision regions $\Omega^{*} \subseteq \Delta_{|\mathcal{H}|}$. From (6), the weighted $\operatorname{cost} \mathcal{C}_{k}$ can be expressed as an inner product,

$$
\mathcal{C}_{k}\left(i_{k}, \mu_{k}\right)=\mu_{k}\left(i_{k}\right)^{\top} \cdot f_{c}\left(s_{k}, \hat{\pi}_{k-1}\right),
$$

where $f_{c}: \mathcal{S} \times \Delta_{|\mathcal{H}|} \rightarrow \mathbb{R}_{+}^{\left|D_{k}\right|}$ is a deterministic vector function that follows from rewriting (6) and $\mu_{k}\left(i_{k}\right)^{\top}$ is a vector given by

$$
\mu_{k}\left(i_{k}\right)^{\top}=\left[P_{D_{k} \mid I_{k}}\left(d_{\mathrm{r}, \mathrm{d}} \mid i_{k}\right), \cdots, P_{D_{k} \mid I_{k}}\left(d_{\mathrm{r}, \mathrm{c}} \mid i_{k}\right)\right] .
$$

The optimal strategy sequence for the finite-horizon, denoted by $\mu_{\mathcal{K}}^{*}:=\left[\mu_{1}^{*}, \ldots, \mu_{N}^{*}\right]$, that minimizes the expected cumulative cost can be obtained by solving the optimization problem given by

$$
\mu_{\mathcal{K}}^{*}=\underset{\mu_{1}^{N} \in \mathcal{U}_{1}^{N}}{\operatorname{argmin}}\left\{\mathbb{E}_{\mu_{1}^{N}}\left[\sum_{k=1}^{N} \mathcal{C}_{k}\left(i_{k}, \mu_{k}\right)\right]\right\} .
$$

According to [12], the optimal strategy $\mu_{k}^{*}$ at each $k \in \mathcal{K}$ can be obtained by solving the backward recursive equation of the stochastic Bellman's dynamic programming given by

$$
V_{k}\left(i_{k}\right)=\min _{\mu_{k} \in \mathcal{U}_{k}}\left\{\mathcal{C}_{k}\left(i_{k}, \mu_{k}\right)+\mathbb{E}_{\mu_{k}}\left[V_{k+1} \mid I_{k}=i_{k}\right]\right\} .
$$

Here $V_{k}$ denotes the value function which is the minimum aggregate expected cost from $k$ to $N$ due to the optimal EMU strategy sequence $\left[\mu_{k}^{*}, \cdots, \mu_{N}^{*}\right]$, initialized with $V_{N+1}$. Further, we have

$$
\begin{gathered}
\mathbb{E}_{\mu_{k}}\left[V_{k+1} \mid I_{k}=i_{k}\right]=\sum_{d_{k}, i_{k+1}} V_{k+1}\left(i_{k+1}\right) P_{D_{k} \mid I_{k}}\left(d_{k} \mid i_{k}\right) \times \\
P_{Z_{k+1} \mid Z_{k}, D_{k}}\left(z_{k+1} \mid z_{k}, d_{k}\right) P_{X_{k+1} \mid H_{k+1}}\left(x_{k+1} \mid h_{k+1}\right) \times \\
P_{H_{k+1} \mid H_{k}}\left(h_{k+1} \mid h_{k}\right) P_{Y_{k} \mid X_{k}, D_{k}}\left(y_{k} \mid x_{k}, d_{k}\right) \\
=: \mu_{k}\left(i_{k}\right)^{\top} \cdot f_{V}\left(V_{k+1}, s_{k}, k\right)
\end{gathered}
$$

where $f_{V}: \mathcal{B}\left(\mathcal{I}_{k+1}\right) \times \mathcal{S} \times \mathcal{K} \rightarrow \mathbb{R}_{+}^{\left|D_{k}\right|}$ is a deterministic vector function. As a direct consequence of (8), (11), and (12), we have that $\left(S_{k}, \hat{\Pi}_{k-1}\right)$ forms a sufficient statistic for the computation of the optimal strategy $\mu_{k}^{*}$ which can be obtained by solving the recursive equation (11) rewritten as

$$
V_{k}\left(s_{k}, \hat{\pi}_{k-1}\right)=\min _{\delta_{k} \in \Delta_{\left|D_{k}\right|}}\left\{\delta_{k}^{\top} \cdot\left[\alpha_{k}\left(s_{k}, \hat{\pi}_{k-1}, V_{k+1}\right)\right]\right\} .
$$

Here $\delta_{k}$ is a vector which represents the conditional probability of control actions of the EMU strategy for a given sufficient statistic $\left(s_{k}, \hat{\pi}_{k-1}\right)$ and $\alpha_{k}(\cdot)$ is a vector function given by

$\alpha_{k}\left(s_{k}, \hat{\pi}_{k-1}, V_{k+1}\right)=f_{c}\left(s_{k}, \hat{\pi}_{k-1}\right)+f_{V}\left(V_{k+1}, s_{k}, k\right)$.
Since the optimization domain of (13) is a simplex, its a linear programming problem and hence there exists an optimal sequence of non-randomized control actions. Further, since $\hat{\pi}_{k-1}$ lies in a continuous space $\Delta_{|\mathcal{H}|}$, in principle, the optimization problem in (13) needs to be solved over an uncountable set. However, similar to the finite dimensional characterization of the value function in [11], exact solution to (13) can be obtained recursively by solving a finite number of linear programming problems obtained by incrementally partitioning the belief space. Algorithm 1 summarizes the approach to obtain the exact optimal solution $\mu_{k}^{*}$.

\section{NUMERICAL STUDY}

To evaluate the optimal EMU strategy we perform numerical simulations of the system model in MATLAB R2018a. In the dynamic programming backward recursion of the optimization problem (13), the optimal solutions are obtained using the Gurobi [13] solver. In the simulations, we design the EMU to hide the occupancy information of the user from an ESS-unaware adversary using the Bayesian hypothesis testing framework [11], where the hypothesis state is binary, i.e., $H_{k}=1$ when the user is absent and $H_{k}=2$ when the user is present. Further, we choose the reference signal to be equal to the user demand in the previous time slot such that the EMU minimizes the variations in the resulting SM readings. We assign the Bayesian cost as $C_{11}=C_{22}=1$ and $C_{12}=C_{21}=0$ such that the Bayesian risk corresponds to the average adversarial detection probability. The simulations were performed using the labelled occupancy data and SM readings from the ECO dataset [14] corresponding to 5 household between June 2012 and January 2013. We assume that the adversary has access to the complete dataset and uses the data of all users to estimate HMM $\hat{\theta}$. To simplify the simulations, we use data from the dataset averaged with 1 hour time resolution and $100 \mathrm{~W}$ power resolution to compute the maximum-likelihood estimate of a non-homogeneous HMM $\theta$ and a homogeneous HMM $\hat{\theta}$. Further, to perform the simulations until the end-oflife of the ESS, we synthesized validation data of 4000 days by randomly selecting the daily data in the dataset with uniform distribution. Further, to reduce the computational complexity of solving the optimal EMU strategies, we divide a day into 6 equal intervals $\Delta_{m}$, where $m \in[1,6]$ and assume that the

$\overline{\text { Algorithm } 1 \text { Exact belief space partitioning algorithm to }}$ obtain the optimal control strategy.

Initialize with a set of polyhedral partitions $\mathcal{G}_{N+1}$ and a piecewise constant function $V_{N+1}$. For $k=N$ to 1, follow: 1: Find $\mathcal{J}_{k+1}$, the set of intersections of $\mathcal{G}_{k+1}$ and the adversarial decision regions $\Omega^{*}$.

2: Find $U_{k}$, the set of inverse linear images of $\mathcal{J}_{k+1}$ under $f_{\hat{\pi}}$, for all $y_{k} \in \mathcal{Y}$.

3: Find $G_{k}$, the set of intersections of $U_{k}$ and $\Delta_{|\mathcal{H}|}$ and construct $\alpha_{k}$ vector function.

4: Solve for $V_{k}$ and $\mu_{k}^{*}, \forall\left(s_{k}, l\right) \in \mathcal{S} \times\left|G_{k}\right|$. 
adversary resets the belief state $\hat{\pi}_{k}$ at the beginning of each of the interval $\Delta_{m}$. This assumption is reasonable as the EMU tries to control the Bayesian risk by driving the adversarial belief state to the partitions in the adversarial decision region using the SM measurements which often aren't explained by the HMM $\hat{\theta}$. That is, for sequence of optimal control actions, the likelihood of the SM measurements given the HMM $\hat{\theta}$ are often very low. This simplification allows us to compute the EMU strategies which are optimal within the whole day time-horizon without significantly increasing the number of the belief space partitions.

Further, in the simulations, we use a lithium-ion battery pack of $48 \mathrm{~V}$ with $1 \mathrm{~kW}$ rated power converter. As in [11], the conditional distribution of a memoryless stochastic ESS model $P_{Z_{k+1}, E_{k}, B_{k} \mid D_{k}, Z_{k}}$ is estimated using the data obtained from the Monte Carlo sampling of the electrochemical models developed in [15], [16]. Further, an average ESS cost function due to the losses $E_{k}$ and $B_{k}$ is obtained by taking the energy cost in Sweden [17] to be $0.2 € / \mathrm{kWh}$ and the lithium-ion battery cost to be $130 € / \mathrm{kWh}$ [18]. In the simulations, we assume that the battery reaches its end of life when $20 \%$ of its rated capacity is lost. Further, the scaling factors of the objective functions $\beta, \gamma$ in the weighted cost function are set, as in [19], by computing the nadir and utopian points of the multi-objective optimization problem.

First, we present the EMU control actions corresponding to optimal single-objective control strategies without trade-off corresponding to user 2 in the ECO dataset. When using a 48V 50Ah lithium-ion battery system Fig. 2 shows the performance of the EMU when tested with an optimal strategy designed to minimize only the Bayesian risk, i.e., using $\omega=0, \sigma=1$. We can see that the EMU schedules the ESS charge and discharge operations that result in user-like energy demand pattens with peaks at times differing from the true peak consumptions. The Bayesian risk is computed by averaging the adversarial detection performance over each interval $\Delta_{m}$, which shows that the control strategy results in low detection probability during the morning time. The observed peaks in the Bayesian risk plot can be explained by the periodic adversarial belief state resets at each interval $\Delta_{m}$. We observe that this strategy increases the variations in the resulting SM readings. On the other hand, the EMU designed to minimize only the variations in the SM readings, i.e., using $\omega=1, \sigma=1$, schedules the charge and discharge operations that result in the flat SM readings over a certain time interval, as shown in Fig. 3. Due to the finite capacity of the ESS, this strategy results in the SM readings switching levels between high and low levels in charge and discharge cycles.

Next, we evaluate the optimal trade-off between the SM variations and the Bayesian risk using $\omega \in[0,1]$ without considering the ESS usage cost in the EMU design by taking $\sigma=1$. Fig. 4 shows the root mean square deviation (RMSD) of the SM readings, average Bayesian risk, ESS lifetime, and the ESS usage cost at different ESS capacities. As expected, we can observe that the minimum RMSD and the minimum achievable Bayesian risk decrease when the ESS capacity

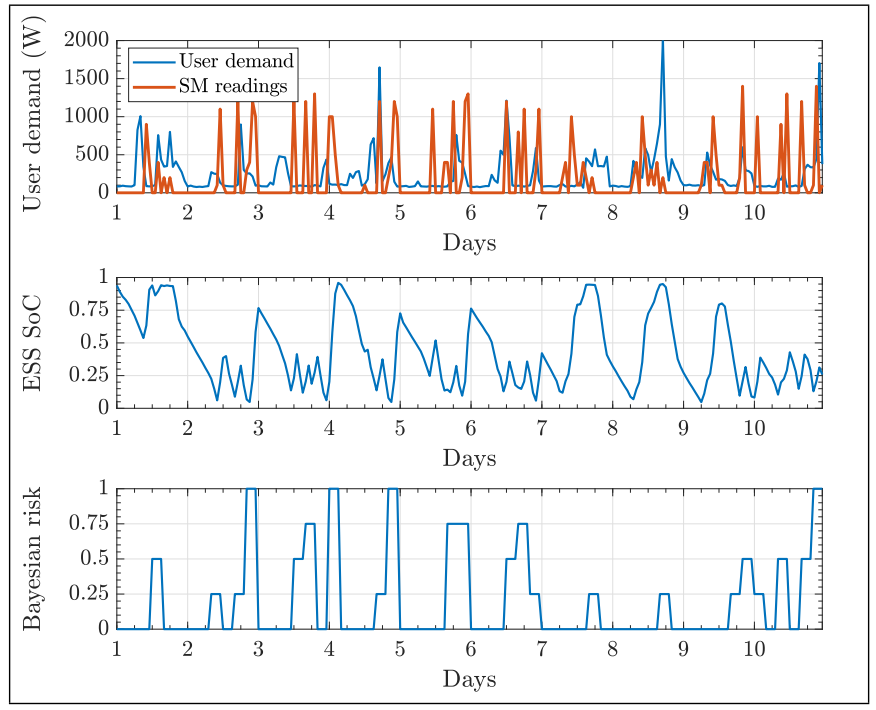

Fig. 2. Hourly user demand, SM readings, and the Bayesian risk when using a 50Ah ESS and the EMU is designed to minimize only the Bayesian risk.

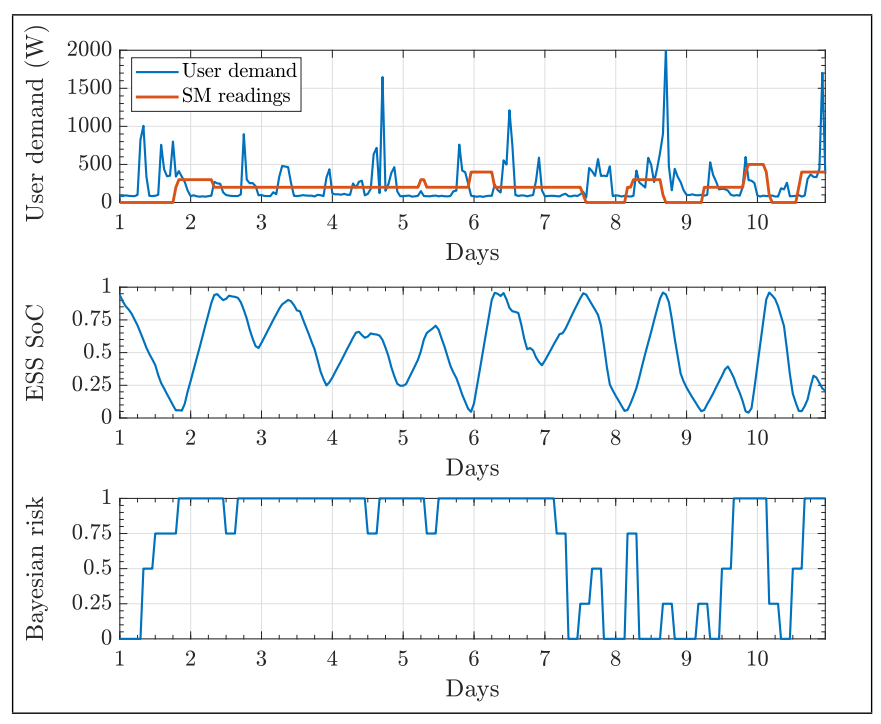

Fig. 3. Hourly user demand, SM readings, and the Bayesian risk when using a 50Ah ESS and the EMU is designed to minimize SM variations.

increases. We can see that, by trading-off some privacy using the trade-off parameter $\omega$, the RMSD of the SM readings can be reduced. Further, we observe that the battery lifetime achieves maximum at $\omega=0.4$ for the simulated system model. Further, by varying the ESS usage cost trade-off parameter, as shown in Fig. 5, we can see that the ESS achieves maximum lifetime when $\sigma=0$, the minimum Bayesian risk is achieved when $(\omega, \sigma)=(0,1)$, and the minimum RMSD of SM measurements when $(\omega, \sigma)=(1,1)$. From the results, we can see that the Bayesian risk and the RMSD behave as opposing objectives and there exists a clear trade-off between the two. Further, for each $\sigma>0$, the battery lifetime achieves maximum at $\omega=0.4$. From the simulation results, we observe that at a fixed ESS capacity, there exists some $\omega^{\#} \in(0,1)$ where the battery lifetime can be maximized while providing some privacy and RMSD of SM measurements. 


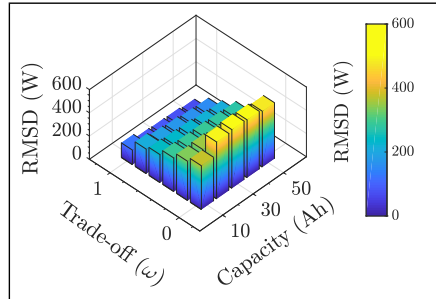

(a) RMSD of SM readings

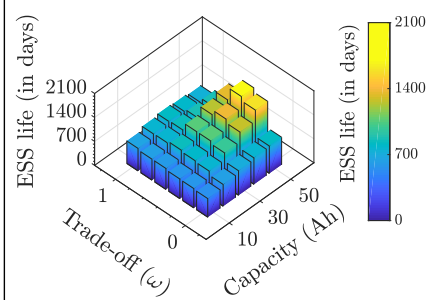

(c) ESS life (in days)

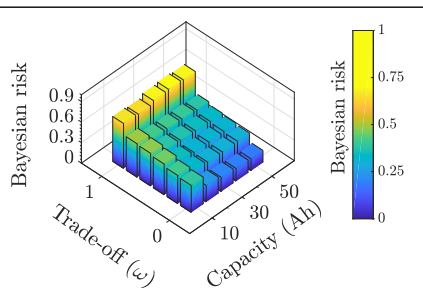

(b) Average Bayesian risk

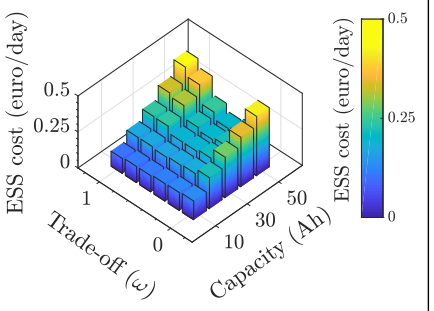

(d) Avg. ESS usage cost
Fig. 4. Pareto-optimal trade-off between the variations in the SM readings and the Bayesian risk at different capacities without considering ESS cost.

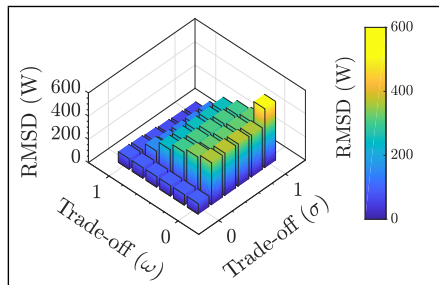

(a) RMSD of SM readings

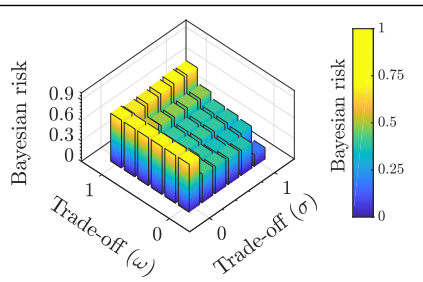

(b) Average Bayesian risk

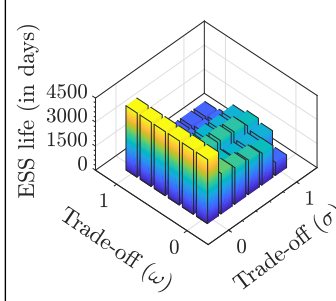

(c) ESS life (in days)
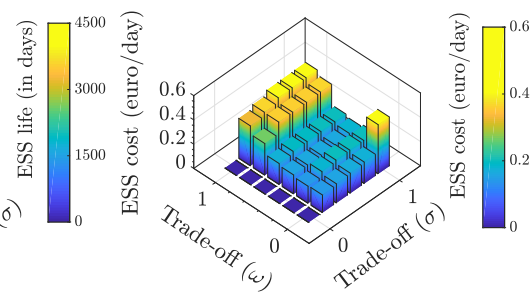

(d) Avg. ESS usage cost
Fig. 5. Pareto-optimal trade-off between the variations in the SM readings and the Bayesian risk while considering the ESS usage cost of 50Ah battery.

\section{CONCLUSiON}

In this paper, we have presented an optimal privacy-bydesign strategy that achieves user demand shaping while considering the ESS usage cost, which is obtained by solving a multi-objective optimization problem using the Markov decision process framework. By measuring the privacy leakage in terms of the Bayesian risk, we show that the dynamic programming recursive equation of the optimization problem need to be solved over a continuous space. Using the finitedimensional structure of the value function, we present an algorithm to compute the exact solution without any approximations using linear programming that partition the belief space recursively. However, the number of linear problems to be solved may increase exponentially with the length of the time-horizon. To limit this exponential complexity in- crease, heuristic control strategies based on the exact-solution algorithm can be used where the belief space partitions are clustered based on the $\alpha$-vectors and spatial proximity of the partitions in the belief space. With extensive numerical simulations using real household consumption data, we show that the designed EMU strategy can be used to optimally tradeoff some privacy and reduce the variations in the SM readings while improving the battery lifetime. In the future work, we plan to study the feasibility of integrating a privacy objective with energy arbitrage in dynamic pricing electricity markets.

\section{REFERENCES}

[1] A. Zoha, A. Gluhak, M. A. Imran, and S. Rajasegarar, "Non-intrusive load monitoring approaches for disaggregated energy sensing: A survey," Sensors, vol. 12, no. 12, pp. 16838-16866, 2012.

[2] G. Giaconi, D. Gunduz, and H. V. Poor, "Privacy-aware smart metering: Progress and challenges," IEEE Signal Processing Magazine, vol. 35, no. 6, pp. 59-78, 2018.

[3] G. Kalogridis, C. Efthymiou, S. Z. Denic, T. A. Lewis, and R. Cepeda, "Privacy for smart meters: Towards undetectable appliance load signatures," in Proc. 1st IEEE Int. Conf. on Smart Grid Commun. (SmartGridComm), Gaithersburg, MD, USA, 2010, pp. 232-237.

[4] S. McLaughlin, P. McDaniel, and W. Aiello, "Protecting consumer privacy from electric load monitoring," in Proc. of the 18th ACM conf. on Computer and comm. security, 2011.

[5] W. Yang, N. Li, Y. Qi, W. Qardaji, S. McLaughlin, and P. McDaniel, "Minimizing private data disclosures in the smart grid," in Proc. of the 19th ACM conf. on Computer and comm. security, 2012.

[6] G. Kalogridis, R. Cepeda, S. Z. Denic, T. Lewis, and C. Efthymiou, "Elecprivacy: Evaluating the privacy protection of electricity management algorithms," IEEE Trans. on Smart Grid, vol. 2, no. 4, 2011.

[7] Z. Li, T. J. Oechtering, and M. Skoglund, "Privacy-preserving energy flow control in smart grids," in Proc. IEEE Int. Conf. on Acoust. Speech Signal Process. (ICASSP), Shanghai, PR China, 2016, pp. 2194-2198.

[8] O. Tan, J. Gómez-Vilardebó, and D. Gündüz, "Privacy-cost tradeoffs in demand-side management with storage," IEEE Transactions on Information Forensics and Security, vol. 12, no. 6, pp. 1458-1469, 2017.

[9] R. R. Avula, T. J. Oechtering, and D. Månsson, "Privacy-preserving smart meter control strategy including energy storage losses," ISGTEurope, Sarajevo, Bosnia and Herzegovina, 2018.

[10] R. R. Avula, T. J. Oechtering, J.-X. Chin, and G. Hug, "Smart meter privacy control strategy including energy storage degradation," in 2019 IEEE Milan PowerTech. IEEE, 2019, pp. 1-6.

[11] R. R. Avula, J.-X. Chin, T. J. Oechtering, G. Hug, and D. Månsson, "Framework for smart meter privacy control strategy design with energy storage limitations," 2019, submitted to IEEE Trans. on smart grid. [Online]. Available: https://kth.box.com/v/framework-sm-privacy

[12] V. Krishnamurthy, Partially Observed Markov Decision Processes. Cambridge University Press, 2016.

[13] L. Gurobi Optimization, "Gurobi optimizer reference manual," 2018. [Online]. Available: http://www.gurobi.com

[14] C. Beckel, W. Kleiminger, R. Cicchetti, T. Staake, and S. Santini, "The eco data set and the performance of non-intrusive load monitoring algorithms," in Proc. 1st ACM Int. Conf. on Embedded Sys. for EnergyEff. Buildings (BuildSys), Memphis, TN, USA, 2014, pp. 80-89.

[15] P. Ramadass, B. Haran, P. M. Gomadam, R. White, and B. N. Popov, "Development of first principles capacity fade model for li-ion cells," Jour. of the Elec.chem. Soc., vol. 151, no. 2, pp. A196-A203, 2004.

[16] H. Ekström and G. Lindbergh, "A model for predicting capacity fade due to sei formation in a commercial graphite/lifepo4 cell," Journal of The Electrochemical Society, vol. 162, no. 6, pp. A1003-A1007, 2015.

[17] Eurostat and U. européenne. Commission européenne, Energy, transport and environment indicators. Office for Official Publications of the European Communities, 2017.

[18] G. Berckmans, M. Messagie, J. Smekens, N. Omar, L. Vanhaverbeke, and J. Van Mierlo, "Cost projection of state of the art lithium-ion batteries for electric vehicles up to 2030," Energies, 2017.

[19] I. Y. Kim and O. L. de Weck, "Adaptive weighted-sum method for bi-objective optimization: Pareto front generation," Structural and multidisciplinary optimization, vol. 29, no. 2, pp. 149-158, 2005. 